\title{
CONVERGENCE ANALYSIS OF THE LDG METHOD FOR SINGULARLY PERTURBED TWO-POINT BOUNDARY VALUE PROBLEMS*
}

\author{
HUIQING ZHU ${ }^{\dagger}$, HAIYAN TIAN ${ }^{\ddagger}$, AND ZHIMIN ZHANG§
}

\begin{abstract}
In this paper the local discontinuous Galerkin method (LDG) is considered for solving one-dimensional singularly perturbed two-point boundary value problems of convection-diffusion type and reaction-diffusion type. Error estimates are studied on Shishkin meshes. The $L^{2}$ error bounds for the LDG approximation of the solution and its derivative are uniformly valid with respect to the singular perturbation parameter. Numerical experiments indicate that the orders of convergence are sharp.
\end{abstract}

Key words. Local discontinuous Galerkin method, singularly perturbed, Shishkin mesh.

AMS subject classifications. 65L11, 65N30, 65N15.

\section{Introduction}

The discontinuous Galerkin (DG) method was introduced in 1973 as a way of solving the steady-state neutron transport equation [16]. Successively in 1974, Lesaint and Raviart made the first analysis for the linear advection equation [12]. Since then many DG methods were vigorously studied. Among these is the local discontinuous Galerkin (LDG) method, which was proposed in [3] and [9] by separating higher order operators into systems of first order equations so that classical DG methods can be extended to problems with second order operators, especially for convection-diffusion and hyperbolic equations. The state of the art of the development of these methods and their applications can be found in $[1,2,5,7,10]$.

In this work, we apply the LDG method to 1-D singularly perturbed problems with Dirichlet boundary conditions:

(1) Convection-diffusion-reaction problem

$$
\begin{aligned}
-\epsilon u^{\prime \prime}+a u^{\prime}+b u & =f & & \text { in } \mathcal{I}=(0,1), \\
u & =0 & & \text { on } \partial \mathcal{I}=\{0,1\},
\end{aligned}
$$

where $0<\epsilon \ll 1$ is the diffusion parameter, $a=a(x) \geq \alpha>0$ accounts for the convection, and $b=b(x)$ accounts for the reaction term. The function $f=f(x)$ is a given source term. We assume that $a, b$, and $f$ are sufficiently smooth on $\overline{\mathcal{I}}$ and satisfy

$$
\begin{array}{ll} 
& b-a^{\prime} / 2 \geq c_{0}>0, \\
\text { or } \quad & b=0, a=\text { constant }
\end{array}
$$

where $\alpha$ and $c_{0}$ are constants independent of $\epsilon$ and mesh size.

\footnotetext{
${ }^{*}$ Received: October 27, 2010; accepted (in revised version): February 22, 2011. Communicated by Shi Jin.

${ }^{\dagger}$ Department of Mathematics, University of Southern Mississippi, Hattiesburg, MS 39406, USA (Huiqing.Zhu@usm.edu).

${ }^{\ddagger}$ Department of Mathematics, University of Southern Mississippi, Hattiesburg, MS 39406, USA (Haiyan.Tian@usm.edu).

$\S$ Department of Mathematics, Wayne State University, Detroit, MI 48202, USA (zzhang@math. wayne.edu).
} 
(2) Reaction-diffusion problem

$$
\begin{aligned}
-\epsilon u^{\prime \prime}+b u & =f & & \text { in } \mathcal{I}=(0,1), \\
u & =0 & & \text { on } \partial \mathcal{I}=\{0,1\} .
\end{aligned}
$$

Here $0<\epsilon \ll \beta^{2} \leq b(x)$ for some constant $\beta$. We assume that $b$ is sufficiently smooth on the domain $\overline{\mathcal{I}}$.

It is well-known that if the standard $h$-version finite element method (FEM), or the similar central difference method, is used in regions where layers occur, then unphysical oscillations arise. This behavior is caused by a small interval of width $O(\epsilon)$ or $O(\sqrt{\epsilon})$ (so-called boundary layer) in which $u^{\prime \prime}$ rapidly changes. Since the solution of the standard FEM exhibits large nonphysical oscillation, many stabilization techniques have been suggested, including upwind, streamline-diffusion FEMs, discontinuous Galerkin methods, and least-squares FEMs [13, 14, 17, 20]. Another effective way for globally solving this problem is to construct a numerical scheme on a layer adapted mesh, such as Shishkin type meshes and Bakhvalov type meshes. There are plenty of theoretical results about FEMs and stabilized FEMs on layer adapted meshes [6, 17, 23]. Recently, the LDG method was considered [20, 22] for numerically solving singularly perturbed problems on layer adapted meshes. Optimal convergence and superconvergence of the LDG solution were observed. There are few analytical results in the literature which have reported a uniform convergence of the DG methods for singularly perturbed problems by using layer adapted meshes (see [18, 21, 24]).

The aim of this paper is to derive $L^{2}$ error estimates of the LDG approximation to the solution and its derivative on a Shishkin mesh for problem (1.1) and problem (1.3). Using numerical traces and interpolation operators similar to those in [8], we prove a uniform convergence rate $O\left((\ln N / N)^{k+1}\right)$ of the LDG approximation to the solution and its derivative, when polynomials of degree no more than $k$ are used. This convergence rate is nearly optimal up to a factor $(\ln N)^{k+1}$. Here "uniform convergence" means that the convergence is uniformly valid with respect to the singular perturbation parameter $\epsilon$. Numerical tests indicate that this convergence rate is sharp and the logarithm factor is not removable. This convergence analysis improves the result listed in [24], where a non-uniform convergence rate was shown for the singularly perturbed convection-diffusion problems by using the LDG method and a Shishkin mesh with a different transition number.

An outline of the paper is as follows. In Section 2, we introduce the LDG discretization. In Section 3, we define layer adapted meshes and state our main results. The proof of main results and related lemmas are provided in Section 4. In Section 5 , numerical experiments are presented to verify the uniform convergence rates.

Notations. Throughout this article, $C$ denotes a generic constant which might not be the same in each appearance. It might depend on the coefficient functions $a$, $b$, the right-hand side function $f$, and the polynomial degree $k$, but is independent of the singular perturbation parameter $\epsilon$, the mesh number $N$, and the mesh sizes $h$ and $H$.

\section{The LDG discretization}

In this section, we first introduce the LDG method we will use to approximate the solutions of problem (1.1) and problem (1.3). The LDG discretization is defined uniformly on an arbitrary mesh, in which the convection term vanishes when discussing the reaction-diffusion problem (1.3). 
We begin by partitioning the domain $\mathcal{I}$. If $0=x_{0}<x_{1}<\ldots<x_{N-1}<x_{N}=1$, we denote by $\mathcal{I}_{N}=\left\{I_{j}=\left(x_{j-1}, x_{j}\right), j=1,2, \cdots, N\right\}$ a partition of domain $\mathcal{I}$, and by $h_{j}=$ $x_{j}-x_{j-1}$ the length of the $j$-th element. Define $v\left(x_{j}^{ \pm}\right)=\lim _{\delta \rightarrow 0} v(x \pm \delta)$ as in [11]. For each element $I_{j} \in \mathcal{I}_{N}$, we set its outward unit normal $n_{I_{j}}\left(x_{j}\right)=1$ and $n_{I_{j}}\left(x_{j-1}\right)=$ -1 . We denote $v_{j}=v\left(x_{j}\right), v_{j}^{ \pm}=v\left(x_{j}^{ \pm}\right), \llbracket v_{0} \rrbracket=-v_{0}^{+}, \llbracket v_{N} \rrbracket=v_{N}^{-}$, and $\llbracket v_{j} \rrbracket=v_{j}^{-} n_{I_{j}}\left(x_{j}\right)+$ $v_{j}^{+} n_{I_{j+1}}\left(x_{j}\right)=v_{j}^{-}-v_{j}^{+}$for $j=1, \cdots, N-1$. $V_{N}$ denotes the finite dimensional space of functions that are polynomial of degree at most $k$ on each element. For any $\mathcal{D} \subseteq \mathcal{I}_{N}$ the Sobolev seminorm on $H^{s}(\mathcal{D})$ is defined as

$$
|v|_{s, \mathcal{D}}:=\left(v^{(s)}, v^{(s)}\right)_{\mathcal{D}}^{1 / 2} .
$$

Accordingly, the Sobolev norm on $H^{r}(\mathcal{D})$ is defined as

$$
\|v\|_{r, \mathcal{D}}:=\left(\sum_{s=0}^{r}|v|_{s, D}^{2}\right)^{1 / 2} .
$$

We drop the first subscript whenever $r=0$, and the second one if $\mathcal{D}=\mathcal{I}_{N}$.

Let $q=\epsilon u^{\prime}$; we rewrite the problems (1.1) and (1.3) as

$$
\begin{aligned}
q & =\epsilon u^{\prime} & & \text { in } \mathcal{I}=(0,1), \\
-q^{\prime}+a u^{\prime}+b u & =f & & \text { in } \mathcal{I}=(0,1), \\
u & =0 & & \text { on } \partial \mathcal{I}=\{0,1\} .
\end{aligned}
$$

We will search for an approximate solution $(Q, U)$ of the LDG method in a finitedimensional subspace of $H^{1}\left(\mathcal{I}_{N}\right) \times H^{1}\left(\mathcal{I}_{N}\right), V_{N} \times V_{N}$. We consider the following problem (see [5]): Find $(Q, U) \in V_{N} \times V_{N}$, such that

$$
\begin{aligned}
& (Q, w)_{\mathcal{I}_{N}}=-\epsilon\left(U, w^{\prime}\right)_{\mathcal{I}_{N}}+\left\langle\epsilon \hat{u}^{\epsilon}, w\right\rangle_{\partial \mathcal{I}_{N}}, \\
& \left(Q-a U, v^{\prime}\right)_{\mathcal{I}_{N}}-\langle\hat{q}, v\rangle_{\partial \mathcal{I}_{N}}+\left\langle a \hat{u}^{c}, v\right\rangle_{\partial \mathcal{I}_{N}}+\left(\left(b-a^{\prime}\right) U, v\right)_{\mathcal{I}_{N}}=(f, v)_{\mathcal{I}_{N}},
\end{aligned}
$$

for all $(w, v) \in V_{N} \times V_{N}$. Here we have used the notations

$$
(\varphi, \psi)_{\mathcal{I}_{N}}=\sum_{I_{j} \in \mathcal{I}_{N}}(\varphi, \psi)_{I_{j}}=\sum_{I_{j} \in \mathcal{I}_{N}} \int_{I_{j}} \varphi(x) \psi(x) d x
$$

and

$$
\langle\varphi, \psi\rangle_{\partial \mathcal{I}_{N}}=\sum_{I_{j} \in \mathcal{I}_{N}}\langle\varphi, \psi\rangle_{\partial I_{j}}=\sum_{j=1}^{N}\left[\varphi_{j}^{-} \psi_{j}^{-}-\varphi_{j-1}^{+} \psi_{j-1}^{+}\right] .
$$

For simplicity, we always write the above two inner products as $(\varphi, \phi)$ and $\langle\varphi, \psi\rangle$ without subscripts for any $\varphi, \phi \in H^{1}\left(\mathcal{I}_{N}\right)$.

To completely define the LDG scheme, it remains to define numerical traces at nodal points. In this article, we take the following numerical traces:

$$
\begin{aligned}
\hat{q}\left(x_{j}\right) & =Q_{j}^{+}-\lambda_{j} \llbracket U_{j} \rrbracket, & & \text { for } j=0,1, \cdots, N-1, \\
\hat{q}(1) & =Q\left(1^{-}\right)-\lambda_{N} U\left(1^{-}\right), & & \\
\hat{u}^{\epsilon}\left(x_{j}\right) & =U_{j}^{-}, & & \text {for } j=1, \cdots, N-1, \\
\hat{u}^{\epsilon}(0) & =0 ; \hat{u}^{\epsilon}(1)=0, & & \\
\hat{u}^{c}\left(x_{j}\right) & =U_{j}^{-}, & & \text {for } j=1, \cdots, N, \\
\hat{u}^{c}(0) & =0, & &
\end{aligned}
$$


where $\lambda_{j} \geq 0(j=0,1, \cdots, N)$ will be determined later. Substituting the numerical traces (2.3) into (2.2), we have

$$
\begin{aligned}
& \epsilon^{-1}(Q, w)+\left(U, w^{\prime}\right)-\sum_{j=1}^{N-1} U_{j}^{-} \llbracket w_{j} \rrbracket=0, \\
& \left(Q-a U, v^{\prime}\right)+\left(\left(b-a^{\prime}\right) U, v\right)+\left(Q_{0}^{+}+\lambda_{0} U_{0}^{+}\right) v_{0}^{+}-\sum_{j=1}^{N-1}\left(Q_{j}^{+}-\lambda_{j} \llbracket U_{j} \rrbracket\right) \llbracket v_{j} \rrbracket \\
& \quad-\left(Q_{N}^{-}-\lambda_{N} U_{N}^{-}\right) v_{N}^{-}+\sum_{j=1}^{N} a_{j}^{-} U_{j}^{-} \llbracket v_{j} \rrbracket=(f, v) .
\end{aligned}
$$

To describe the structure of the LDG scheme clearly, we define some bilinear forms:

$$
\begin{aligned}
a(Q, w) & =\epsilon^{-1}(Q, w), \\
b_{1}(U, w) & =\left(U, w^{\prime}\right)-\sum_{j=1}^{N-1} U_{j}^{-} \llbracket w_{j} \rrbracket, \\
b_{2}(Q, v) & =\left(Q, v^{\prime}\right)-\sum_{j=0}^{N-1} Q_{j}^{+} \llbracket v_{j} \rrbracket-Q_{N}^{-} v_{N}^{-}, \\
c(U, v) & =-\left(a U, v^{\prime}\right)+\left(\left(b-a^{\prime}\right) U, v\right)+\sum_{j=1}^{N} a_{j}^{-} U_{j}^{-} \llbracket v_{j} \rrbracket+\sum_{j=0}^{N} \lambda_{j} \llbracket U_{j} \rrbracket \llbracket v_{j} \rrbracket, \\
f(v) & =(f, v) .
\end{aligned}
$$

In this article we assume that $a_{j}^{+}=a_{j}^{-}=a_{j}$ for any $j=0,1, \cdots, N$. Using integration by parts, we can verify that $b_{1}(v, w)=-b_{2}(w, v)$. As a consequence, $(2.4)$ can be written into a system

$$
\begin{aligned}
a(Q, w)+b_{1}(U, w) & =0, \\
-b_{1}(v, Q)+c(U, v) & =f(v) .
\end{aligned}
$$

REMARK 2.1. Although the auxiliary variable $q:=\epsilon u^{\prime}$, we actually used $\epsilon^{-1} q:=u^{\prime}$ in (2.6). Thus, the LDG formulation remains symmetric since $\epsilon$ appears only in $a(Q, w)$. It is possible to define the auxiliary variable $q:=u^{\prime}$ or $q:=\sqrt{\epsilon} u^{\prime}$. These two different choices have no impact on the mesh and the numerical results since the generation of the mesh depends only on the regularity of $u$. However, if we use them, more caution is needed in doing theoretical analysis because there are many terms containing $\epsilon$, which would be mixed up easily.

The following proposition guarantees the existence and uniqueness of the numerical solution defined by (2.2) and (2.3).

Proposition 2.2. If $\lambda_{j} \geq 0(j=0,1, \cdots, N)$, then the $L D G$ solution determined by (2.2) and numerical traces (2.3) exists and is unique.

Proof. We only need to verify that $Q=0, U=0$ in (2.6) if $f=0$. Taking $w=Q$ and $v=U, f=0$ in (2.6), and adding (2.6a)and (2.6b) together we get

$$
\begin{aligned}
& a(Q, Q)+c(U, U) \\
= & \epsilon^{-1}\|Q\|_{\mathcal{I}_{N}}^{2}-\left(a U, U^{\prime}\right)+\left(\left(b-a^{\prime}\right) U, U\right)+\sum_{j=1}^{N} a_{j} U_{j}^{-} \llbracket U_{j} \rrbracket+\sum_{j=0}^{N} \lambda_{j} \llbracket U_{j} \rrbracket^{2} \\
= & 0 .
\end{aligned}
$$


By an integration by parts,

$$
-\left(a U, U^{\prime}\right)=\left(a U, U^{\prime}\right)+\left(a^{\prime} U, U\right)-\sum_{j=1}^{N}\left[a_{j}\left(U_{j}^{-}\right)^{2}-a_{j-1}\left(U_{j-1}^{+}\right)^{2}\right] .
$$

Solving the above equation for $\left(a U, U^{\prime}\right)$ one has

$$
-\left(a U, U^{\prime}\right)=\frac{1}{2}\left(a^{\prime} U, U\right)-\frac{1}{2}\left[-a_{0}\left(U_{0}^{+}\right)^{2}+\sum_{j=1}^{N-1} a_{j} \llbracket U_{j}^{2} \rrbracket+a_{N}\left(U_{N}^{-}\right)^{2}\right] .
$$

Therefore, the sum of the second term, the third term, and the fourth term of (2.7) can be simplified as

$$
\begin{aligned}
& \left(\left(b-a^{\prime} / 2\right) U, U\right)+\frac{1}{2} a_{0}\left(U_{0}^{+}\right)^{2}-\frac{1}{2} a_{N}\left(U_{N}^{-}\right)^{2} \\
& +\sum_{j=1}^{N-1} a_{j}\left[\left(U_{j}^{-}\right)^{2}-U_{j}^{-} U_{j}^{+}-\frac{1}{2}\left(U_{j}^{-}\right)^{2}+\frac{1}{2}\left(U_{j}^{+}\right)^{2}\right]+a_{N}\left(U_{N}^{-}\right)^{2} \\
= & \left\|\left(b-a^{\prime} / 2\right)^{\frac{1}{2}} U\right\|_{\mathcal{I}_{N}}^{2}+\sum_{j=0}^{N} \frac{1}{2} a_{j} \llbracket U_{j} \rrbracket^{2} .
\end{aligned}
$$

By substituting (2.9) into (2.7), the left side of (2.7) becomes

$$
a(Q, Q)+c(U, U)=\epsilon^{-1}\|Q\|_{\mathcal{I}_{N}}+\left\|\left(b-a^{\prime} / 2\right)^{\frac{1}{2}} U\right\|_{\mathcal{I}_{N}}^{2}+\sum_{j=0}^{N}\left(\frac{1}{2} a_{j}+\lambda_{j}\right) \llbracket U_{j} \rrbracket^{2},
$$

which implies that

$$
\begin{aligned}
U=0, Q & =0 & & \text { if }(1.2 \mathrm{a}), \\
U \in C^{0}(\mathcal{I}), Q & =0 & & \text { if }(1.2 \mathrm{~b}) .
\end{aligned}
$$

When $(1.2 \mathrm{~b})$ holds true, using an integration by parts for $(2.5 \mathrm{~b})$ we write $(2.6 \mathrm{a})$ as

$$
\epsilon^{-1}(Q, w)-\left(U^{\prime}, w\right)-U_{0}^{+} w_{0}^{+}+U_{N}^{-} w_{N}^{-}+\sum_{j=1}^{N-1} \llbracket U_{j} \rrbracket w_{j}^{+}=0 .
$$

It follows from $(2.11 \mathrm{~b})$ that $\left(U^{\prime}, w\right)=0$ for all $w \in V_{N}$, which implies that $U$ is a piecewise constant function on $\overline{\mathcal{I}}$ by taking $w=U^{\prime}$. This, together with the implementation of $(2.11 \mathrm{~b})$, proves that $U=0$. The existence and uniqueness of the LDG solution follow as a consequence.

Define the compact form of our LDG discretization as

$$
\mathcal{A}(\phi, \psi ; w, v)=a(\phi, w)+b_{1}(\psi, w)+b_{2}(\phi, v)+c(\psi, v),
$$

for any $(\phi, \psi),(w, v) \in V_{N} \times V_{N}$. Based on (2.10), the compact form (2.12) introduces a norm

$$
|(w, v)|_{\mathcal{A}}^{2}:=\mathcal{A}(w, v ; w, v)=\epsilon^{-1}\|w\|_{\mathcal{I}_{N}}^{2}+\left\|\left(b-a^{\prime} / 2\right)^{\frac{1}{2}} v\right\|_{\mathcal{I}_{N}}^{2}+\sum_{j=0}^{N}\left(\frac{1}{2} a_{j}+\lambda_{j}\right) \llbracket v_{j} \rrbracket^{2}
$$


for any $(w, v) \in V_{N} \times V_{N}$.

By the consistency of the numerical traces, it is straightforward to verify the orthogonality

$$
\mathcal{A}(q-Q, u-U ; w, v)=0
$$

for any $(w, v) \in V_{N} \times V_{N}$.

\section{Shishkin meshes and main results}

We begin this section by defining our layer adapted meshes, i.e., Shishkin meshes. Then we state our main results of the LDG method on Shishkin meshes. The proofs will be provided in Section 4 .

Shishkin meshes type 1: For convection-diffusion problem (1.1).

The exact solution of (1.1) admits a decomposition of the form

$$
u=\bar{u}+u_{\epsilon},
$$

where the component functions have the regularity $[15,23]$

$$
\left|\bar{u}^{(j)}(x)\right| \leq C \quad \text { and } \quad\left|u_{\epsilon}^{(j)}(x)\right| \leq C \epsilon^{-j} e^{-\alpha(1-x) / \epsilon}
$$

for all $x$ in $\mathcal{I}$ and $j=0,1, \cdots, k+2$. Consequently, the decomposition of $q$ is in the form $q=\bar{q}+q_{\epsilon}=\epsilon \bar{u}^{\prime}+\epsilon u_{\epsilon}^{\prime}$ with regularity

$$
\left|\bar{q}^{(j)}(x)\right| \leq C \epsilon, \quad\left|q_{\epsilon}^{(j)}(x)\right| \leq C \epsilon^{-j} e^{-\alpha(1-x) / \epsilon}
$$

for any $x$ in $\mathcal{I}$ and $j=0,1, \cdots, k+1$.

We now define a Shishkin mesh that is appropriate for the problem (1.1). Let $N \geq 2$ be a multiple of 2 . Define the transition point number

$$
\tau=\min \left\{\frac{1}{2}, \frac{k+1}{\alpha} \epsilon \ln N\right\}
$$

and set

$$
H=2 \frac{1-\tau}{N} \quad \text { and } \quad h=2 \frac{\tau}{N} .
$$

The nodes of the mesh $\mathcal{I}_{N}$ are defined recursively by setting $x_{0}=0$ and

$$
x_{j}= \begin{cases}x_{j-1}+H, & \text { for } j=1,2, \ldots, N / 2, \\ x_{j-1}+h, & \text { for } j=N / 2+1, N / 2+2, \ldots, N .\end{cases}
$$

Since the mesh $\mathcal{I}_{N}$ is piecewise uniform we define

$$
\mathcal{I}_{\mathrm{R}}=\bigcup_{j=1}^{N / 2} I_{j} \quad \text { and } \quad \mathcal{I}_{\mathrm{BL}}=\bigcup_{j=N / 2+1}^{N} I_{j}
$$

Clearly, $\mathcal{I}_{\mathrm{R}}$ is a uniform discretization of the interval $(0,1-\tau)$ of meshsize $H$, and $\mathcal{I}_{\mathrm{BL}}$ is that of the interval $(1-\tau, 1)$ of meshsize $h$. 
Shishkin meshes type 2: For reaction-diffusion problem (1.3).

The exact solution of the (1.3) admits a decomposition of the form

$$
u=\bar{u}+u_{\epsilon, 1}+u_{\epsilon, 2},
$$

where the component functions have the regularity $[15,23]$

$$
\begin{aligned}
& \left|\bar{u}^{(j)}(x)\right| \leq C, \quad\left|u_{\epsilon, 1}^{(j)}(x)\right| \leq C \epsilon^{-j / 2} e^{-\beta x / \sqrt{\epsilon}}, \\
& \left|u_{\epsilon, 2}^{(j)}(x)\right| \leq C \epsilon^{-j / 2} e^{-\beta(1-x) / \sqrt{\epsilon}}
\end{aligned}
$$

for any $x$ in $\mathcal{I}$ and $j=0,1, \cdots, k+2$. The components of $q=\bar{q}+q_{\epsilon, 1}+q_{\epsilon, 2}=\epsilon \bar{u}^{\prime}+\epsilon u_{\epsilon, 1}^{\prime}+$ $\epsilon u_{\epsilon, 2}^{\prime}$ satisfy

$$
\begin{aligned}
\left\|\bar{q}^{(i)}\right\|_{\infty, \mathcal{I}} & \leq C \epsilon, \quad\left|q_{\epsilon, 1}^{(j)}(x)\right| \leq C \epsilon^{-(j-1) / 2} e^{-\beta x / \sqrt{\epsilon}}, \\
\left|q_{\epsilon, 2}^{(j)}(x)\right| & \leq C \epsilon^{-(j-1) / 2} e^{-\beta(1-x) / \sqrt{\epsilon}}
\end{aligned}
$$

for any $x$ in $\mathcal{I}$ and $j=0,1, \cdots, k+1$.

We now define a Shishkin mesh that is appropriate for the problem (1.3). Let $N \geq 4$ be a multiple of 4 . Let

$$
\tau=\min \left\{\frac{1}{4}, \frac{k+1}{\beta} \sqrt{\epsilon} \ln N\right\}
$$

and set

$$
H=2 \frac{(1-2 \tau)}{N} \text { and } \quad h=\frac{4 \tau}{N} .
$$

The nodes of the mesh $\mathcal{I}_{N}$ are defined recursively by setting $x_{0}=0$ and

$$
x_{j}= \begin{cases}x_{j-1}+h, & \text { for } j=1,2, \ldots, N / 4, \\ x_{j-1}+H, & \text { for } j=N / 4+1, \ldots, 3 N / 4, \\ x_{j-1}+h, & \text { for } j=3 N / 4+1, \ldots, N .\end{cases}
$$

Since the mesh $\mathcal{I}_{N}$ is piecewise uniform we define

$$
\mathcal{I}_{\mathrm{R}}=\bigcup_{j=N / 4+1}^{3 N / 4} I_{j} \quad \text { and } \quad \mathcal{I}_{\mathrm{BL}}=\mathcal{I} \backslash \mathcal{I}_{\mathrm{R}} .
$$

Clearly, $\mathcal{I}_{\mathrm{R}}$ is a uniform discretization of the interval $(\tau, 1-\tau)$ of meshsize $H$, and $\mathcal{I}_{\mathrm{BL}}$ is that of the interval $(0, \tau) \cup(1-\tau, 1)$ of meshsize $h$.

For the two types of Shishkin meshes, let us note that $1 / N \leq H \leq 2 / N$, and hence there exists a constant $C \in(1 / 2,1)$ such that $H=C / N$.

We are now ready to state out main results. The first theorem is about the error estimates for the LDG solution of the convection-diffusion problem (1.1).

Theorem 3.1. If $\lambda_{j}=0$ for all $j=0,1, \cdots, N-1$ and $\lambda_{N} \geq 0$, then there exists a constant $C$ independent of $\epsilon$ and $N$ such that

$$
\sqrt{\epsilon}\left\|u^{\prime}-\epsilon^{-1} Q\right\|+\left\|\left(b-a^{\prime}\right)^{\frac{1}{2}}(u-U)\right\| \leq C\left(\frac{\ln N}{N}\right)^{k+1},
$$


and

$$
|(q-Q, u-U)|_{\mathcal{A}} \leq C\left(\frac{\ln N}{N}\right)^{k+\frac{1}{2}}
$$

We will make some remarks before we state next theorem.

REMARK 3.2. Comparing with the LDG method for non-singular problems considered in [5], we see that the error bound in (3.7) is nearly optimal up to a factor $(\ln N)^{k+1}$. It has been shown in [23] that the finite element method on Shishkin meshes achieves a superconvergence rate that is the same as (3.7).

REMARK 3.3. Error bounds in (3.7) and (3.8) are uniformly valid in terms of the singular perturbation parameter $\epsilon$ since they are independent of $\epsilon$. It was point out in [21] that the the central point of global error estimates for singularly perturbed problems is the $\epsilon$ independence. The author also gave a brief review of many results in the literature, which have norms of the exact solution appearing in error bounds.

REMARK 3.4. The LDG method was considered for solving singularly perturbed convection-diffusion problem with Cauchy boundary conditions in [21]. The boundary conditions were taken such that the problem can be separated into two first-order differential equations. Then a uniform convergence rate $\sqrt{\epsilon}(\ln N / N)^{k+1}$ was established under the $L^{2}$-norm for a first-order differential equation. However, this technique cannot be applied to Dirichlet boundary conditions.

Our next result is the error estimate for the LDG solution of the reaction-diffusion problem (1.3).

THEOREM 3.5. If $\lambda_{j}=0$ for all $j=0,1, \cdots, N-1$ and $\lambda_{N} \geq 0$, then there exists a constant $C$ independent of $\epsilon$ and $N$ such that

$$
\begin{aligned}
\sqrt{\epsilon}\left\|u^{\prime}-\epsilon^{-1} Q\right\|+\|u-U\| & \leq C\left[\sqrt[4]{\epsilon}\left(\frac{\ln N}{N}\right)^{k+1}+\frac{1}{N^{k+1}}\right], \\
|(q-Q, u-U)|_{\mathcal{A}} & \leq C\left(\frac{\ln N}{N}\right)^{k+\frac{1}{2}}
\end{aligned}
$$

Moreover, if $u_{\mathrm{R}} \in V_{N}$, then we have

$$
\sqrt{\epsilon}\left\|u^{\prime}-\epsilon^{-1} Q\right\|+\|u-U\| \leq C\left[\sqrt[4]{\epsilon}\left(\frac{\ln N}{N}\right)^{k+1}+\frac{1}{N^{k+\frac{3}{2}}}\right] .
$$

Remark 3.6. Note that in [23], the finite element method on Shishkin meshes was also applied to the reaction-diffusion problem and achieved the same convergence rate, where the error was measured by a discrete $\epsilon$-weighted norm. As a corollary, a convergence rate of the error at mesh points was proved, although it is not optimal. In Theorem 3.1 and Theorem 3.5, the convergence rates are established for the $L^{2}$-norm. The pointwise error estimate of the LDG approximation for both problems (1.1) and (1.3) is an ongoing work.

REMARK 3.7. If the parameters $\left\{\lambda_{j}\right\}_{0}^{N}$ take different values, then the convergence rates may be inferior to those in Theorem 3.1 and Theorem 3.5, as shown at the end of Section 4.4. 
REMARK 3.8. The factor $\sqrt[4]{\epsilon}$ is from the interpolation error estimates of $u_{\epsilon}$ and $q_{\epsilon}$. This factor cannot be removed by adjusting the transition number of the Shishkin mesh. However, it may not be observed in the numerical experiments when $\bar{u}$ is not in the finite element space since the other term $N^{k+1}$ of (3.9) will be dominated if $\epsilon \ll 1$. These results are the same as Theorem 2.2 [23], where the error of the FEM solution was bounded in a discrete energy norm.

REMARK 3.9. The analysis of this work could be extended to the Bakhvalov type meshes. The difficulty of the extension is in the interpolation error estimates. So far there is no theoretical result in the literature about the uniform convergence of discontinuous Galerkin methods on Bakhvalov type meshes.

\section{Proofs}

This section is devoted to the proof of the main results stated in Section 3. A detailed proof for Theorem 3.1 is given. Since the proof of Theorem 3.5 is very similar, we only sketch its proof in Section 4.2.

4.1. Proof of Theorem 3.1. To provide a detailed proof for Theorem 3.1, we proceed in several steps. First, we define projection type interpolants and introduce some approximation properties. In Step 2 we prove some interpolation error estimates. Step 3 is the main step in which we estimate the errors between the interpolations and the numerical solutions. Finally, in Step 4, we combine the estimates of Step 2 and Step 3 to prove Theorem 3.1.

Step 1: Interpolations. We use polynomial interpolation of degree $k \geq 0$. Let $I=$ $\left(a^{+}, a^{-}\right)$be an arbitrary interval and $\mathcal{P}^{k}(I)$ the space of polynomials of degree no more than $k$ on $I$.

For $v \in C(\bar{I})$, we define the projection $\pi^{ \pm} v \in \mathcal{P}^{k}(I)$ by using the following two conditions:

$$
\pi^{ \pm} v\left(a^{ \pm}\right)=v\left(a^{ \pm}\right), \quad \int_{I}\left[v(x)-\pi^{ \pm} v(x)\right] p^{\prime}(x) d x=0
$$

for any $p(x) \in \mathcal{P}^{k}(I)$. Define the interpolation of $u$ and $q$ as

$$
\mathbb{I} u_{\left.\right|_{I_{j}}}=\pi^{-} u_{\left.\right|_{I_{j}}}, \quad \mathbb{I} q_{\left.\right|_{I_{j}}}=\pi^{+} q_{\left.\right|_{I_{j}}}
$$

for any $j=1,2, \cdots, N$. Let $\xi_{u}:=u-\mathbb{I} u, \eta_{u}:=\mathbb{I} u-U, e_{u}:=u-U, \xi_{q}:=q-\mathbb{I} q, \eta_{q}:=$ $\mathbb{I} q-Q$, and $e_{q}:=q-Q$. As a consequence, we have $e_{u}=\xi_{u}+\eta_{u}, e_{q}=\xi_{q}+\eta_{q}$.

In the following proof, we will estimate $\left(\xi_{q}, \xi_{u}\right)$ and $\left(\eta_{q}, \eta_{u}\right)$ separately. To estimate the interpolation errors $\xi_{u}$ and $\xi_{q}$, we need two preliminary lemmas and some frequently used inequalities. The first lemma was proven in [19].

Lemma 4.1. (Lemma 3.7, [19]) For any $v \in C(\bar{I})$, the interpolation operators $\pi^{ \pm}$ satisfy

$$
\left\|\pi^{ \pm} v\right\|_{I}^{2} \leq C\left(\|v\|_{I}^{2}+\left|v\left(a^{ \pm}\right)\right|^{2}\right)
$$

on the reference element $I=\left(a^{+}, a^{-}\right)$.

The second lemma gives elementwise error bounds of the interpolation (see [4]). 
LEMma 4.2. (Lemma 3.3, [4]) If $\mathcal{I}_{N}$ is an arbitrary decomposition of the domain $\mathcal{I}$ and $u$ is the exact solution of problem (1.1) (or problem (1.3)), then there exists a constant $C$ such that

$$
\begin{aligned}
\left|\xi_{u}\left(x_{j}^{+}\right)\right| & \leq C h_{j}^{s-\frac{1}{2}}\|u\|_{s, I_{j}}, j=0,1, \cdots, N-1, \\
\left|\xi_{q}\left(x_{j}^{-}\right)\right| & \leq C h_{j}^{s-\frac{1}{2}}\|q\|_{s, I_{j}}, j=1,2, \cdots, N, \\
\left\|\xi_{u}\right\|_{I_{j}} & \leq C h_{j}^{s}\|u\|_{s, I_{j}}, \\
\left\|\xi_{q}\right\|_{I_{j}} & \leq C h_{j}^{s}\|q\|_{s, I_{j}},
\end{aligned}
$$

for any $I_{j} \in \mathcal{I}_{N}(j=1,2, \cdots, N)$ and $s=0,1, \cdots, k+1$.

Two frequently used inequalities:

$$
\begin{aligned}
\sum_{i=0}^{N / 2-1} H e^{-2 \alpha\left(1-x_{i}\right) / \epsilon} & \leq \int_{0}^{1-\tau} e^{-2 \alpha(1-x) / \epsilon} d x \leq C \epsilon N^{-2(k+1)}, \\
e^{-2 \alpha\left(1-x_{N / 2}\right) / \epsilon} & =e^{-2 \alpha \tau / \epsilon} \leq C N^{-2(k+1)}
\end{aligned}
$$

where we used the value of the transition point number (3.3).

Step 2: The estimation of $\left(\xi_{q}, \xi_{u}\right)$.

LEMMA 4.3. Suppose that $u$ is the solution of the convection-diffusion problem (1.1) satisfying regularity (3.1)-(3.2). There exists a constant $C$ such that

$$
\begin{array}{r}
\epsilon^{-1 / 2}\left\|\xi_{q}\right\|_{\mathcal{I}_{N}}+\left\|\xi_{u}\right\|_{\mathcal{I}_{N}} \leq C\left(\frac{\ln N}{N}\right)^{k+1}, \\
\left|\xi_{q}\left(1^{-}\right)\right| \leq C\left(\frac{\ln N}{N}\right)^{k+1}, \\
\left(\sum_{j=0}^{N} \llbracket\left(\xi_{u}\right)_{j} \rrbracket^{2}\right)^{\frac{1}{2}} \leq C\left(\frac{\ln N}{N}\right)^{k+\frac{1}{2}} .
\end{array}
$$

Proof. To prove (4.5a), we estimate each component of the decomposition $u=\bar{u}+u_{\epsilon}$ and $q=\bar{q}+q_{\epsilon}$. (3.1)-(3.2) state that the $(k+1)$-th derivatives of the regular part $\bar{u}$ and $\epsilon^{-1} \bar{q}$ have constant upper bounds. Using (4.3c)-(4.3d) we have

$$
\begin{aligned}
& \left\|\xi_{\bar{q}}\right\|_{\mathcal{I}_{N}} \leq C\left(H^{N+1}+h^{k+1}\right)|\bar{q}|_{H^{k+1}(\mathcal{I})} \leq C \epsilon N^{-(k+1)} \\
& \left\|\xi_{\bar{u}}\right\|_{\mathcal{I}_{N}} \leq C\left(H^{N+1}+h^{k+1}\right)|\bar{u}|_{H^{k+1}(\mathcal{I})} \leq C N^{-(k+1)}
\end{aligned}
$$

Next, we shall estimate the other components $\xi_{u_{\epsilon}}$ and $\xi_{q_{\epsilon}}$ on $\mathcal{I}_{\mathrm{R}}$ and $\mathcal{I}_{\mathrm{BL}}$ separately. Applying (4.3c)-(4.3d) to each element of $\mathcal{I}_{\mathrm{BL}}$ and using the regularity (3.1)-(3.2) one obtains

$$
\begin{aligned}
\left\|\xi_{q_{\epsilon}}\right\|_{\mathcal{I}_{\mathrm{BL}}}+\left\|\xi_{u_{\epsilon}}\right\|_{\mathcal{I}_{\mathrm{BL}}} & \leq C h^{k+1}\left(\left|q_{\epsilon}\right|_{H^{k+1}\left(\mathcal{I}_{\mathrm{BL}}\right)}+\left|u_{\epsilon}\right|_{H^{k+1}\left(\mathcal{I}_{\mathrm{BL}}\right)}\right) \\
& \leq C h^{k+1}\left[\int_{1-\tau}^{1} \epsilon^{-2(k+1)} e^{\frac{-2 \alpha(1-x)}{\epsilon}} d x\right]^{\frac{1}{2}} \\
& \leq C \sqrt{\epsilon}\left(\frac{\ln N}{N}\right)^{k+1}
\end{aligned}
$$


where we used the fact that $\mathcal{I}_{\mathrm{BL}}$ is an uniform discretization of the domain $(1-\tau, 1)$ of meshsize $h$.

Consider the estimation on $\mathcal{I}_{R}$. By triangle inequality we get $\left\|\xi_{q_{\epsilon}}\right\|_{\mathcal{I}_{\mathbb{R}}} \leq\left\|q_{\epsilon}\right\|_{\mathcal{I}_{\mathrm{R}}}+$ $\left\|\mathbb{I} q_{\epsilon}\right\|_{\mathcal{I}_{\mathrm{R}}}$. The first term is estimated by combining (4.3d) and (3.2),

$$
\left\|q_{\epsilon}\right\|_{\mathcal{I}_{R}} \leq C\left(\int_{0}^{1-\tau} e^{-2 \alpha(1-x) / \epsilon} d x\right)^{\frac{1}{2}} \leq C \sqrt{\epsilon} N^{-(k+1)} .
$$

Here we used (3.3). Applying (4.2), the scaling argument, and (4.4a) to the second term yields

$$
\begin{aligned}
\left\|I q_{\epsilon}\right\|_{\mathcal{I}_{\mathrm{R}}} & \leq C\left(\left\|q_{\epsilon}\right\|_{\mathcal{I}_{\mathrm{R}}}^{2}+H \sum_{i=0}^{N / 2-1}\left|q_{\epsilon}\left(x_{i}\right)\right|^{2}\right)^{\frac{1}{2}} \\
& \leq C\left(\epsilon N^{-2(k+1)}+H \sum_{i=0}^{N / 2-1} e^{-2 \alpha\left(1-x_{i}\right) / \epsilon}\right)^{\frac{1}{2}} \\
& \leq \sqrt{\epsilon} N^{-(k+1)} .
\end{aligned}
$$

Combining (4.8) with (4.9) establishes the error estimate of $\xi_{q}$ on $\mathcal{I}_{\mathrm{R}}$. Like the above proof, it can be shown that $\xi_{u}$ is bounded by

$$
\left\|\xi_{u_{\epsilon}}\right\|_{\mathcal{I}_{\mathrm{R}}} \leq 2\left(\left\|u_{\epsilon}\right\|_{\mathcal{I}_{\mathrm{R}}}^{2}+\left\|\mathbb{I} u_{\epsilon}\right\|_{\mathcal{I}_{\mathrm{R}}}^{2}\right)^{\frac{1}{2}} \leq C \sqrt{\epsilon+1 / N} N^{-(k+1)}
$$

The result (4.5a) immediately follows from the combination of (4.6)-(4.10).

Next, we consider the interpolation error at nodal points. Using (4.3b) we have

$$
\left|\xi_{q}\left(1^{-}\right)\right| \leq C h^{k+\frac{1}{2}}|q|_{k+1, I_{N}} \leq C h^{k+\frac{1}{2}} \epsilon^{-(k+1)} h^{\frac{1}{2}} \leq C(\ln N / N)^{k+1} .
$$

In view of $(4.1), \llbracket\left(\xi_{u}\right)_{j} \rrbracket^{2}=\left(\xi_{u}\right)_{j}^{+}$for all $j=0,1, \cdots, N-1$ and $\llbracket\left(\xi_{u}\right)_{N} \rrbracket^{2}=0$. By (4.3a) and (3.1),

$$
\sum_{j=N / 2}^{N-1}\left[\left(\xi_{u}\right)_{j}^{+}\right]^{2} \leq C h^{2 k+1}|u|_{k+1, \mathcal{I}_{\mathrm{BL}}}^{2} \leq C(\ln N / N)^{2 k+1} .
$$

Using the decomposition of $u$ and triangle inequality one has

$$
\sum_{j=0}^{N / 2-1}\left[\left(\xi_{u}\right)_{j}^{+}\right]^{2} \leq \sum_{j=0}^{N / 2-1}\left[\left(\xi_{\bar{u}}\right)_{j}^{+}\right]^{2}+\sum_{j=0}^{N / 2-1}\left(u_{\epsilon}\right)_{j}^{2}+\sum_{j=0}^{N / 2-1}\left[\left(\mathbb{I} u_{\epsilon}\right)_{j}^{+}\right]^{2} .
$$

Applying (4.3a) and (3.1) yields an upper bound $C N^{-(2 k+1)}$ for the first term of (4.12). By (3.1) and (4.4a), the second term is bounded by $C \epsilon N^{-(2 k+1)}$. To estimate the third term of (4.12), we need the trace inequality

$$
\left(\mathbb{I} u_{\epsilon}\right)_{j}^{+}=-\int_{x_{j}}^{x_{j}+1}\left(\mathbb{I} u_{\epsilon}\right)^{\prime} d x \leq C H^{\frac{1}{2}}\left\|\left(\mathbb{I} u_{\epsilon}\right)^{\prime}\right\|_{I_{j}} \leq C H^{-\frac{1}{2}}\left\|\mathbb{I} u_{\epsilon}\right\|_{I_{j}}
$$

for $j=0,1, \cdots, x_{N / 2}-1$. Here we have used the inverse inequality to prove the last step. By (4.13) and (4.2), we get

$$
\sum_{j=0}^{N / 2-1}\left[\left(\mathbb{I} u_{\epsilon}\right)_{j}^{+}\right]^{2} \leq C H^{-1}\left\|\mathbb{I} u_{\epsilon}\right\|_{\mathcal{I}_{\mathrm{R}}}^{2} \leq C H^{-1}\left[\left\|u_{\epsilon}\right\|_{\mathcal{I}_{\mathrm{R}}}^{2}+H \sum_{j=0}^{N / 2}\left(u_{\epsilon}\right)_{j}^{2}\right]
$$


which, by (3.1) and (4.4), is bounded by $C(\epsilon+1 / N) N^{-(2 k+1)}$. Combining error bounds of the three terms of (4.12), together with (4.11), yields (4.5c).

Step 3: The boundedness of $\mathcal{A}\left(\xi_{q}, \xi_{u} ; w, v\right)$.

LEMmA 4.4. If $\lambda_{N} \geq 0, \lambda_{j}=0$ for $j=0,1, \cdots, N-1$, then there exists a constant $C$ such that

$$
\mathcal{A}\left(\xi_{q}, \xi_{u} ; w, v\right) \leq C\left(\frac{\ln N}{N}\right)^{k+1}|(w, v)|_{\mathcal{A}}
$$

for any $(w, v) \in V_{N} \times V_{N}$.

Proof. By the definition of the bilinear form $\mathcal{A}(2.12)$, we have

$$
\begin{aligned}
\mathcal{A}\left(\xi_{q}, \xi_{u} ; w, v\right) & =a\left(\xi_{q}, w\right)+b_{1}\left(\xi_{u}, w\right)+b_{2}\left(\xi_{q}, v\right)+c\left(\xi_{u}, v\right) \\
& =S_{1}+S_{2}+S_{3}+S_{4} .
\end{aligned}
$$

We estimate $S_{1}-S_{4}$ individually. First, using Schwarz's inequality and (4.5a) one obtains

$$
S_{1} \leq \epsilon^{-1}\left\|\xi_{q}\right\|_{\mathcal{I}_{N}}\|w\|_{\mathcal{I}_{N}} \leq C\left(\frac{\ln N}{N}\right)^{k+1} \epsilon^{-1 / 2}\|w\|_{\mathcal{I}_{N}} \leq C\left(\frac{\ln N}{N}\right)^{k+1}|(w, v)|_{\mathcal{A}} .
$$

The definition of the interpolation operator $(4.1)$ implies $\left(\xi_{u}, w^{\prime}\right)=\left(\xi_{q}, v^{\prime}\right)=0,\left(\xi_{u}\right)_{j}^{-}=$ 0 for $j=1,2, \cdots, N$ and $\left(\xi_{q}\right)_{j}^{+}=0$ for $j=0,1, \cdots, N-1$. Therefore,

$$
\begin{gathered}
S_{2}=\left(\xi_{u}, w^{\prime}\right)+\sum_{j=1}^{N-1}\left(\xi_{u}\right)_{j}^{-} \llbracket(w)_{j} \rrbracket=0, \\
S_{3}=\left(\xi_{q}, v^{\prime}\right)-\sum_{j=0}^{N-1}\left(\xi_{q}\right)_{j}^{+} \llbracket(v)_{j} \rrbracket-\xi_{q}\left(1^{-}\right) v\left(1^{-}\right)=-\xi_{q}\left(1^{-}\right) v\left(1^{-}\right) .
\end{gathered}
$$

Using error estimate (4.5b) and the definition of the norm (2.13) we get

$$
S_{3} \leq C\left|\xi_{q}\left(1^{-}\right)\right||(w, v)|_{\mathcal{A}} \leq C\left(\frac{\ln N}{N}\right)^{k+1}|(w, v)|_{\mathcal{A}}
$$

Consider $S_{4}$. Let $\bar{a}$ be a piecewise constant function defined by $\left.\bar{a}\right|_{I_{j}}=\frac{1}{h_{j}} \int_{I_{j}} a(x) d x$ for all $j=1,2, \cdots, N$. Therefore, $\left(\xi_{u}, \bar{a} v^{\prime}\right)=0$ and $\|a-\bar{a}\|_{L^{\infty}\left(I_{j}\right)} \leq C h_{j}\|a\|_{W_{\infty}^{1}\left(I_{j}\right)}$ for any $j=1,2, \cdots, N$. Like the proof of $S_{2}$, we use the facts $\left(\xi_{u}\right)_{j}^{-}=0$ for $j=1,2, \cdots, N$ and $\left(\xi_{q}\right)_{j}^{+}=0$ for $j=0,1, \cdots, N-1$ to obtain

$$
S_{4}=\left(\xi_{u},(a-\bar{a}) v^{\prime}\right)-\left(\xi_{u},\left(b-a^{\prime}\right) v\right)+\sum_{j=0}^{N} \lambda_{j} \llbracket\left(\xi_{u}\right)_{j} \rrbracket \llbracket(v)_{j} \rrbracket .
$$

Consider the first two terms of $S_{4}$. If $(1.2 \mathrm{~b})$ holds true, instantly we have $\left(\xi_{u},(a-\right.$ $\left.\bar{a}) v^{\prime}\right)+\left(\xi_{u},\left(b-a^{\prime}\right) v\right)=0$. Otherwise, if $(1.2 \mathrm{a})$, by Schwarz's inequality, the approximation property of $\bar{a}$ and inverse inequality we obtain

$$
\left(\xi_{u},(a-\bar{a}) v^{\prime}\right) \leq C \sum_{j=1}^{N} h_{j}\|a\|_{W_{\infty}^{1}\left(I_{j}\right)} \frac{1}{h_{j}}\left\|\xi_{u}\right\|_{I_{j}}\|v\|_{I_{j}} \leq C\left\|\xi_{u}\right\|_{\mathcal{I}_{N}}\left\|\sqrt{b-a^{\prime} / 2} v\right\|_{\mathcal{I}_{N}}
$$


It is straightforward to prove that the second term of $S_{4}$ is also bounded by $C\left\|\xi_{u}\right\|_{\mathcal{I}_{N}}\left\|\sqrt{b-a^{\prime} / 2} v\right\|_{\mathcal{I}_{N}}$. Here the constant $C$ depends on $b, a^{\prime}$, and $c_{0}$. By (2.13) and Schwarz's inequality, the third term of $S_{4}$ is controlled by $\left(\sum_{j=0}^{N} \lambda_{j} \llbracket\left(\xi_{u}\right)_{j} \rrbracket\right)^{1 / 2}\left(\sum_{j=0}^{N} \lambda_{j} \llbracket v_{j} \rrbracket\right)^{1 / 2}$. Since $\left(\sum_{j=0}^{N} \lambda_{j} \llbracket v_{j} \rrbracket\right)^{1 / 2}$ and $\left\|\sqrt{b-a^{\prime} / 2} v\right\|_{\mathcal{I}_{N}}$ are parts of the $|(w, v)|_{\mathcal{A}}$, we use (4.5) to prove

$$
S_{4} \leq \begin{cases}C\left(\frac{\ln N}{N}\right)^{k+1}|(w, v)|_{\mathcal{A}}, & \text { if } \lambda_{N} \geq 0 ; \lambda_{j}=0, j=0,1, \cdots, N-1 ; \\ C\left(\frac{\ln N}{N}\right)^{k+\frac{1}{2}}|(w, v)|_{\mathcal{A}}, & \text { if } \lambda_{j}=O(1), j=0,1, \cdots, N \\ C\left(\frac{\ln N}{N}\right)^{k}|(w, v)|_{\mathcal{A}} & \text { if } \lambda_{j}=O\left(\frac{\epsilon}{h}\right), j=0,1, \cdots, N .\end{cases}
$$

Collecting the estimates of $S_{1}-S_{4}$ establishes the estimate (4.14).

\section{Step 4: Proof of Theorem 3.1.}

Proof. If we set $(w, v)=\left(\eta_{q}, \eta_{u}\right)$ in the compact form $\mathcal{A}\left(\eta_{q}, \eta_{u} ; w, v\right)$, it follows directly by using the definition of the norm (2.13) and the orthogonality property (2.14) that

$$
\begin{aligned}
\left|\left(\eta_{q}, \eta_{u}\right)\right|_{\mathcal{A}}^{2} & =\mathcal{A}\left(\eta_{q}, \eta_{u} ; \eta_{q}, \eta_{u}\right) \\
& =\mathcal{A}\left(-\xi_{q},-\xi_{u} ; \eta_{q}, \eta_{u}\right)+\mathcal{A}\left(e_{q}, e_{u} ; \eta_{q}, \eta_{u}\right) \\
& =\mathcal{A}\left(-\xi_{q},-\xi_{u} ; \eta_{q}, \eta_{u}\right) \\
& =-\mathcal{A}\left(\xi_{q}, \xi_{u} ; \eta_{q}, \eta_{u}\right) .
\end{aligned}
$$

Therefore, the combination of Lemma 4.3 and Lemma 4.4 produces the error estimate

$$
\left|\left(\eta_{q}, \eta_{u}\right)\right|_{\mathcal{A}} \leq C\left(\frac{\ln N}{N}\right)^{k+1}
$$

for convection-diffusion problem (1.1). Combining (4.18), (4.5a), and (4.5c) we obtain (3.7) and (3.8).

4.2. Proof of Theorem 3.5. Since the proof of Theorem 3.5 is similar to the proof of Theorem 3.1, we only provide the outline of the proof. Like Section 4.1, we proceed in several steps.

Step 1: The estimation of $\left(\xi_{q}, \xi_{u}\right)$. We use the same interpolations as in Section 4.1. The interpolation error bounds for the reaction-diffusion are derived in a similar way. The proof is done by straightforward calculation which is analogous to the proof of Lemma 4.3 .

Lemma 4.5. Suppose that $u$ is the solution of the reaction-diffusion problem (1.3) satisfying regularity (3.4)-(3.5). There exists a constant $C$ such that

$$
\begin{aligned}
\epsilon^{-1 / 2}\left\|\xi_{q}\right\|_{\mathcal{I}_{N}}+\left\|\xi_{u}\right\|_{\mathcal{I}_{N}} & \leq C\left[\sqrt[4]{\epsilon}\left(\frac{\ln N}{N}\right)^{k+1}+\frac{1}{N^{k+1}}\right], \\
\left(\sum_{j=0}^{N} \llbracket\left(\xi_{u}\right)_{j} \rrbracket^{2}\right)^{\frac{1}{2}} \leq C\left(\frac{\ln N}{N}\right)^{k+\frac{1}{2}} & \\
\left|\xi_{q}\left(1^{-}\right)\right| & \leq C \sqrt{\epsilon}\left(\frac{\ln N}{N}\right)^{k+1}
\end{aligned}
$$


Moreover, if the regular part of the solution $\bar{u} \in V_{N}$, there exists a constant $C$ such that

$$
\epsilon^{-1 / 2}\left\|\xi_{q}\right\|_{\mathcal{I}_{N}}+\left\|\xi_{u}\right\|_{\mathcal{I}_{N}} \leq C\left[\sqrt[4]{\epsilon}\left(\frac{\ln N}{N}\right)^{k+1}+\frac{1}{N^{k+\frac{3}{2}}}\right]
$$

Proof. Like the proof of Lemma 4.3, we estimate each component of $u$ and $q$ using similar arguments.

(1) Consider $\left\|\xi_{q}\right\|_{\mathcal{I}_{N}}$ and $\left\|\xi_{u}\right\|_{\mathcal{I}_{N}}$ on $\mathcal{I}_{N}$. The analysis is based on the regularities (3.5)-(3.4). Firstly, we have

$$
\begin{aligned}
\left\|\xi_{\bar{u}}\right\|_{\mathcal{I}_{N}} & \leq C N^{-(k+1)} \\
\left\|\xi_{u_{\epsilon, 1}}\right\|_{\mathcal{I}_{N}} & \leq\left\|\xi_{u_{\epsilon, 1}}\right\|_{(0, \tau)}+\left\|\xi_{u_{\epsilon, 1}}\right\|_{(\tau, 1-\tau)}+\left\|\xi_{u_{\epsilon, 1}}\right\|_{(1-\tau, 1)} .
\end{aligned}
$$

From the scaling arguments, (4.2), (4.3c), and (4.3d), we obtain

$$
\begin{aligned}
\left\|\xi_{u_{\epsilon, 1}}\right\|_{(0, \tau)}^{2} & \leq C h^{2(k+1)}\left\|u_{\epsilon, 1}\right\|_{(0, \tau)}^{2} \\
& \leq C\left(\frac{\sqrt{\epsilon} \ln N}{N}\right)^{2(k+1)} \epsilon^{-(k+1)} \int_{0}^{\tau} e^{-\frac{2 \beta x}{\sqrt{\epsilon}}} d x \leq C \sqrt{\epsilon}\left(\frac{\ln N}{N}\right)^{2(k+1)} \\
\left\|\xi_{u_{\epsilon, 1}}\right\|_{(1-\tau, 1)}^{2} & \leq C h^{2(k+1)}\left\|u_{\epsilon, 1}\right\|_{(1-\tau, 1)} \\
& \leq C\left(\frac{\sqrt{\epsilon} \ln N}{N}\right)^{2(k+1)} \epsilon^{-(k+1)} \int_{1-\tau}^{1} e^{-\frac{2 \beta x}{\sqrt{\epsilon}}} d x \leq C\left(\frac{\sqrt{\epsilon} \ln N}{N}\right)^{2(k+1)} \\
\left\|\xi_{u_{\epsilon, 1}}\right\|_{(\tau, 1-\tau)}^{2} & \leq C\left\|u_{\epsilon, 1}\right\|_{(\tau, 1-\tau)}^{2}+C \sum_{i=N / 4+1}^{3 N / 4} H\left|u_{\epsilon, 1}\left(x_{i}\right)\right|^{2} \\
& \leq C \int_{\tau}^{1-\tau} e^{-\frac{2 \beta x}{\sqrt{\epsilon}}} d x \leq C \sqrt{\epsilon}\left(e^{-\frac{2 \beta(1-\tau)}{\sqrt{\epsilon}}}-e^{-\frac{2 \beta \tau}{\sqrt{\epsilon}}}\right) \leq C \sqrt{\epsilon} N^{-2(k+1)}
\end{aligned}
$$

Combining the above inequalities and (4.21) yields

$$
\left\|\xi_{u_{\epsilon, 1}}\right\|_{\mathcal{I}_{N}} \leq C \sqrt[4]{\epsilon}\left(\frac{\ln N}{N}\right)^{k+1}
$$

By the symmetric regularities of $u_{\epsilon, 1}$ and $u_{\epsilon, 2}$, we use the same technique to obtain

$$
\begin{aligned}
\left\|\xi_{u_{\epsilon, 2}}\right\|_{(0, \tau)}+\left\|\xi_{u_{\epsilon, 2}}\right\|_{(1-\tau, 1)} & \leq C\left(\frac{\sqrt{\epsilon} \ln N}{N}\right)^{k+1}+C \sqrt[4]{\epsilon}\left(\frac{\ln N}{N}\right)^{k+1} \\
\left\|\xi_{u_{\epsilon, 2}}\right\|_{(\tau, 1-\tau)} & \leq C\left(\int_{\tau}^{1-\tau} e^{-\frac{2 \beta x}{\sqrt{\epsilon}}} d x\right)^{1 / 2}+C \sqrt{H}\left|u_{\epsilon, 2}(1-\tau)\right| \\
& \leq C\left(\sqrt[4]{\epsilon}+\frac{1}{\sqrt{N}}\right) N^{-(k+1)}
\end{aligned}
$$

which implies that

$$
\left\|\xi_{u_{\epsilon, 2}}\right\|_{\mathcal{I}_{N}} \leq C\left[\sqrt[4]{\epsilon}\left(\frac{\ln N}{N}\right)^{k+1}+N^{-\left(k+\frac{3}{2}\right)}\right]
$$


The combination of (4.21)-(4.23) yields the upper bound of $\left\|\xi_{u}\right\|_{\mathcal{I}_{N}}$ :

$$
\left\|\xi_{u}\right\|_{\mathcal{I}_{N}} \leq C\left[\sqrt[4]{\epsilon}\left(\frac{\ln N}{N}\right)^{k+1}+N^{-(k+1)}\right] .
$$

By the decomposition of $q=\bar{q}+q_{\epsilon, 1}+q_{\epsilon, 2}=\epsilon \bar{u}+\epsilon u_{\epsilon, 1}+\epsilon u_{\epsilon, 2}$, the analogous analysis can be applied to the estimation of $q$. The detailed proof is omitted. We only list the error bounds for each component of $q$ :

$$
\begin{aligned}
&\left\|\xi_{\bar{q}}\right\|_{\mathcal{I}_{N}} \leq C \epsilon N^{-(k+1)} \\
& \frac{1}{\sqrt{\epsilon}}\left\|\xi_{q_{\epsilon, 1}}\right\|_{\mathcal{I}_{N}} \leq C\left[\sqrt[4]{\epsilon}\left(\frac{\ln N}{N}\right)^{k+1}+N^{-\left(k+\frac{3}{2}\right)}\right], \\
& \frac{1}{\sqrt{\epsilon}}\left\|\xi_{q_{\epsilon, 2}}\right\|_{\mathcal{I}_{N}} \leq C \sqrt[4]{\epsilon}\left(\frac{\ln N}{N}\right)^{k+1} .
\end{aligned}
$$

Thus, the error bound of $\left\|\xi_{q}\right\|_{\mathcal{I}_{N}}$ follows by combining these three error estimates:

$$
\frac{1}{\sqrt{\epsilon}}\left\|\xi_{q}\right\|_{\mathcal{I}_{N}} \leq C\left[\sqrt[4]{\epsilon}\left(\frac{\ln N}{N}\right)^{k+1}+N^{-\left(k+\frac{3}{2}\right)}\right] .
$$

The inequality (4.19a) follows directly from (4.24) and (4.26).

(2) The other two inequalities (4.19b) and (4.19c) can be shown by repeating the proof of (4.5c) and (4.5b) under the assumption of (3.5) and (3.4):

$$
\begin{array}{rlrl}
\left(\sum_{j=0}^{N} \llbracket\left(\xi_{\bar{u}}\right)_{j} \rrbracket^{2}\right)^{\frac{1}{2}} & \leq C\left(\frac{\ln N}{N}\right)^{k+\frac{1}{2}}, \quad\left(\sum_{j=0}^{N} \llbracket\left(\xi_{u_{\epsilon}}\right)_{j} \rrbracket^{2}\right)^{\frac{1}{2}} \leq C\left(\frac{\ln N}{N}\right)^{k+\frac{1}{2}}, \\
\left|\xi_{\bar{q}}\left(1^{-}\right)\right| & \leq C \epsilon^{k+2}\left(\frac{\ln N}{N}\right)^{k+1}, & \left|\xi_{q_{\epsilon}}\left(1^{-}\right)\right| \leq C \sqrt{\epsilon}\left(\frac{\ln N}{N}\right)^{k+1} .
\end{array}
$$

The combination of these error bounds yields (4.19b)-(4.19c). If $\bar{u} \in V_{N}$, we have $\xi_{\bar{u}}=$ $\xi_{\bar{q}}=0$. Therefore, it is straightforward to verify (4.20) by collecting error estimates (4.22), (4.23), and (4.25).

Step 2: The boundedness of $\mathcal{A}\left(\xi_{q}, \xi_{u} ; w, v\right)$. Since the convection term is gone, i.e., $a(x)=0$, this step is almost the same as Step 3 of Section 4.1. The error estimations are in the following lemma. The proof is omitted.

Lemma 4.6. If $\lambda_{N} \geq 0, \lambda_{j}=0$ for $j=0,1, \cdots, N-1$, then there exists a constant $C$ such that

$$
\mathcal{A}\left(\xi_{q}, \xi_{u} ; w, v\right) \leq C\left[\sqrt[4]{\epsilon}\left(\frac{\ln N}{N}\right)^{k+1}+\frac{1}{N^{k+1}}\right]|(w, v)|_{\mathcal{A}} .
$$

Moreover, if $\bar{u} \in V_{N}$, then

$$
\mathcal{A}\left(\xi_{q}, \xi_{u} ; w, v\right) \leq C\left[\sqrt[4]{\epsilon}\left(\frac{\ln N}{N}\right)^{k+1}+\left(\frac{1}{N}\right)^{k+\frac{3}{2}}\right]|(w, v)|_{\mathcal{A}} .
$$


Step 3: Proof of Theorem 3.5. Repeating the analysis of the error $\left(\eta_{q}, \eta_{u}\right)$ in the Step 4 of Section 4.1 we have

$$
\left|\left(\eta_{q}, \eta_{u}\right)\right|_{\mathcal{A}} \leq C\left(\frac{\ln N}{N}\right)^{k+1}
$$

and if the regular part of the solution $\bar{u} \in V_{N}$,

$$
\left|\left(\eta_{q}, \eta_{u}\right)\right|_{\mathcal{A}} \leq C\left[\sqrt[4]{\epsilon}\left(\frac{\ln N}{N}\right)^{k+1}+\left(\frac{1}{N}\right)^{k+\frac{3}{2}}\right] .
$$

Combining (4.27) and (4.19) we obtain (3.9). (3.10) directly follows (4.28) and (4.20).

\section{Numerical results}

In this section, we display numerical results to verify our theoretical results. We consider three test problems. In order to observe the order of convergence of the error, at each refinement of the mesh, we compute the approximate order of convergence as follows. Define the $\epsilon$-seminorm of the error $\left(e_{q}, e_{u}\right)$ as

$$
\left|\left(e_{q}, e_{u}\right)\right|_{\epsilon}:=\epsilon^{-\frac{1}{2}}\left\|e_{q}\right\|_{\mathcal{I}_{N}}+\left\|e_{u}\right\|_{\mathcal{I}_{N}} .
$$

Let $\left|\left(e_{q}, e_{u}\right)\right|_{\epsilon}^{N}$ denote the error of approximation using a mesh with $N$ elements, then

$$
\log _{p}\left(\frac{\left|\left(e_{q}, e_{u}\right)\right|_{\epsilon}^{N}}{\left|\left(e_{q}, e_{u}\right)\right|_{\epsilon}^{2 N}}\right)
$$

with base $p=2(\ln N / \ln 2 N)$ is the approximate order of convergence. This is a reflection of the fact that, due to Theorem 3.1 and Theorem 3.5, we are expecting a convergence with respect to a power of $\ln N / N$. We display this quantity in the columns of Table 5.1-5.3 labeled "order".

Test Problem 1. We consider convection-diffusion problem (1.1) with $a(x)=e^{-x}$, $b(x)=1$ such that the exact solution $u(x)$ is

$$
u(x)=x\left(\frac{x}{2}+\epsilon\right)-\frac{\left(\frac{1}{2}+\epsilon\right)\left(e^{(x-1) / \epsilon}-e^{-1 / \epsilon}\right)}{1-e^{-1 / \epsilon}} .
$$

Let the stabilization parameter $\lambda_{j}=0$ for $j=0,1, \cdots, N$. From Table 5.1, we see that the error of $\left|\left(e_{q}, e_{u}\right)\right|_{\epsilon}$ indeed converges uniformly with order $(\ln N / N)^{k+1}$ as predicted by Theorem 3.1. Since Theorem 3.1 states that convergence rates are independent of the parameter $\epsilon$, the errors for different $\epsilon$ values will be very close, which is confirmed by numerical results in Table 5.1. The errors are plotted in Figure 5.1, in which we denote by $E_{k}(k=1,2,3)$ the $L^{2}$ errors when using a piecewise polynomial space of degree $k(k=1,2,3)$.

Test Problem 2. We consider convection-diffusion problem (1.1) with $a(x)=1$, $b(x)=0$, and $f(x)=\sin \pi x$ such that the exact solution $u(x)$ is

$$
u(x)=\frac{1+e^{-1 / \epsilon}-2 e^{-(1-x) / \epsilon}}{\pi\left(1+\pi^{2} \epsilon^{2}\right)\left(1-e^{-1 / \epsilon}\right)}+\frac{\epsilon \pi \sin \pi x-\cos \pi x}{\pi\left(1+\pi^{2} \epsilon^{2}\right)} .
$$

Let the stabilization parameter $\lambda_{j}=0$ for $j=0,1, \cdots, N$. The data in Table 5.2 verifies the theoretical results of Theorem 3.1. The errors are plotted in Figure 5.2. 


\begin{tabular}{|c|c|c|c|c|c|c|}
\hline & \multicolumn{6}{|c|}{$\epsilon=10^{-4}$} \\
\hline & \multicolumn{2}{|c|}{$k=1$} & \multicolumn{2}{|c|}{$k=2$} & \multicolumn{2}{|c|}{$k=3$} \\
\hline$N$ & error & order & error & order & error & order \\
\hline 32 & $3.86 \mathrm{e}-03$ & - & $4.43 \mathrm{e}-04$ & - & $5.46 \mathrm{e}-05$ & - \\
\hline 64 & $1.42 \mathrm{e}-03$ & 1.96 & $9.89 \mathrm{e}-05$ & 2.94 & $7.43 \mathrm{e}-06$ & 3.91 \\
\hline 128 & $4.87 \mathrm{e}-04$ & 1.98 & $1.99 \mathrm{e}-05$ & 2.97 & $8.79 \mathrm{e}-07$ & 3.96 \\
\hline 256 & $1.60 \mathrm{e}-04$ & 1.99 & $3.75 \mathrm{e}-06$ & 2.99 & $9.47 \mathrm{e}-08$ & 3.98 \\
\hline \multirow[t]{3}{*}{512} & $5.09 \mathrm{e}-05$ & 1.99 & $6.71 \mathrm{e}-07$ & 2.99 & $9.53 \mathrm{e}-09$ & 3.99 \\
\hline & \multicolumn{6}{|c|}{$\epsilon=10^{-8}$} \\
\hline & \multicolumn{2}{|c|}{$k=1$} & \multicolumn{2}{|c|}{$k=2$} & \multicolumn{2}{|c|}{$k=3$} \\
\hline$N$ & error & order & error & order & error & order \\
\hline 32 & $3.86 \mathrm{e}-03$ & - & $4.42 \mathrm{e}-04$ & - & $5.46 \mathrm{e}-05$ & - \\
\hline 64 & $1.46 \mathrm{e}-03$ & 1.96 & $9.88 \mathrm{e}-05$ & 2.93 & $7.42 \mathrm{e}-06$ & 3.91 \\
\hline 128 & $4.87 \mathrm{e}-04$ & 1.98 & $1.99 \mathrm{e}-05$ & 2.97 & $8.79 \mathrm{e}-07$ & 3.96 \\
\hline 256 & $1.60 \mathrm{e}-04$ & 1.99 & $3.75 \mathrm{e}-06$ & 2.98 & $9.45 \mathrm{e}-08$ & 3.98 \\
\hline 512 & $5.09 \mathrm{e}-05$ & 1.99 & $6.71 \mathrm{e}-07$ & 2.99 & $9.47 \mathrm{e}-09$ & 4.00 \\
\hline
\end{tabular}

TABle 5.1. History of convergence for $\left|\left(e_{q}, e_{u}\right)\right|_{\epsilon}$ for Test Problem 1. $\tau=(k+1) \epsilon \ln N$.
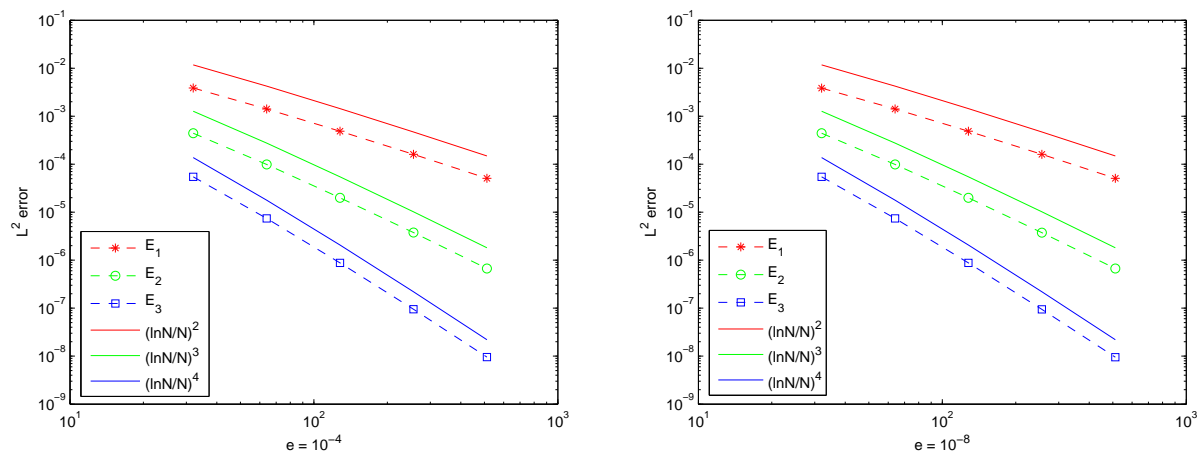

FIG. 5.1. The LDG method for Test Problem 1 with $k=1,2,3, \epsilon=10^{-4}, 10^{-8}$.
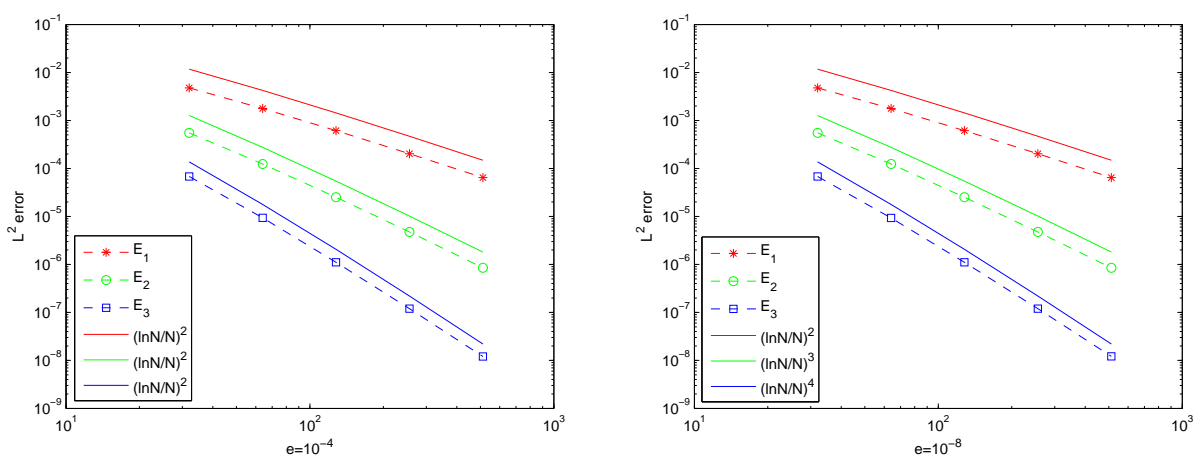

FIG. 5.2. The LDG method for Test Problem 2 with $k=1,2,3, \epsilon=10^{-4}, 10^{-8}$. 


\begin{tabular}{|c|c|c|c|c|c|c|}
\hline & \multicolumn{6}{|c|}{$\epsilon=10^{-4}$} \\
\hline & \multicolumn{2}{|c|}{$k=1$} & \multicolumn{2}{|c|}{$k=2$} & \multicolumn{2}{|c|}{$k=3$} \\
\hline$N$ & error & order & error & order & error & order \\
\hline 32 & $4.77 \mathrm{e}-03$ & - & $5.51 \mathrm{e}-04$ & - & $6.81 \mathrm{e}-05$ & - \\
\hline 64 & $1.77 \mathrm{e}-03$ & 1.94 & $1.24 \mathrm{e}-04$ & 2.92 & $9.33 \mathrm{e}-06$ & 3.89 \\
\hline 128 & $6.14 \mathrm{e}-04$ & 1.97 & $2.52 \mathrm{e}-05$ & 2.96 & $1.11 \mathrm{e}-06$ & 3.95 \\
\hline 256 & $2.03 \mathrm{e}-04$ & 1.98 & $4.75 \mathrm{e}-06$ & 2.98 & $1.20 \mathrm{e}-07$ & 3.98 \\
\hline \multirow[t]{3}{*}{512} & $6.46 \mathrm{e}-05$ & 1.99 & $8.52 \mathrm{e}-07$ & 2.99 & $1.21 \mathrm{e}-08$ & 3.99 \\
\hline & \multicolumn{6}{|c|}{$\epsilon=10^{-8}$} \\
\hline & \multicolumn{2}{|c|}{$k=1$} & \multicolumn{2}{|c|}{$k=2$} & \multicolumn{2}{|c|}{$k=3$} \\
\hline$N$ & error & order & error & order & error & order \\
\hline 32 & $4.77 \mathrm{e}-03$ & - & $5.51 \mathrm{e}-04$ & - & $6.81 \mathrm{e}-05$ & - \\
\hline 64 & $1.77 \mathrm{e}-03$ & 1.94 & $1.24 \mathrm{e}-04$ & 2.92 & $9.33 \mathrm{e}-06$ & 3.89 \\
\hline 128 & $6.14 \mathrm{e}-04$ & 1.97 & $2.52 \mathrm{e}-05$ & 2.96 & $1.11 \mathrm{e}-06$ & 3.95 \\
\hline 256 & $2.03 \mathrm{e}-04$ & 1.98 & $4.75 \mathrm{e}-06$ & 2.98 & $1.20 \mathrm{e}-07$ & 3.98 \\
\hline 512 & $6.46 \mathrm{e}-05$ & 1.99 & $8.52 \mathrm{e}-07$ & 2.99 & $1.21 \mathrm{e}-08$ & 3.98 \\
\hline
\end{tabular}

TABle 5.2. History of convergence for $\left|\left(e_{q}, e_{u}\right)\right|_{\epsilon}$ for Test Problem 2. $\tau=(k+1) \epsilon \ln N$.

Test Problem 3. We apply the LDG method to reaction-diffusion problem (1.3) with $b(x)=1$, and $f(x)=1$ such that the exact solution $u(x)$ is

$$
u(x)=1-\frac{e^{-x / \sqrt{\epsilon}}+e^{(x-1) / \sqrt{\epsilon}}}{1+e^{-1 / \sqrt{\epsilon}}} .
$$

It is clear that the regular part $\bar{u}$ of the exact solution is in the approximation space $V_{N}$. Let the value of the stabilization parameter $\lambda_{j}=0$ for $j=0,1, \cdots, N-1$, and $\lambda_{N}=$ $k / h$. Table 5.3 indicates that the error of $\left|\left(e_{q}, e_{u}\right)\right|_{\epsilon}$ converges with order $(\ln N / N)^{k+1}$. Comparing errors for different values of $\epsilon$, is is easy see the presence of the factor $\sqrt[4]{\epsilon}$. Table 5.3 also indicates that the term $\sqrt[4]{\epsilon}(\ln N / N)^{k+1}$ in (3.10) dominates the convergence rate of the error. The reason is that the second term $N^{-(k+3 / 2)}$ of the error bound (3.10) is smaller than the dominating term when $\bar{u} \in V_{N}$, as predicted by Theorem 3.5. All errors of Table 5.3 are plotted in Figure 5.3.
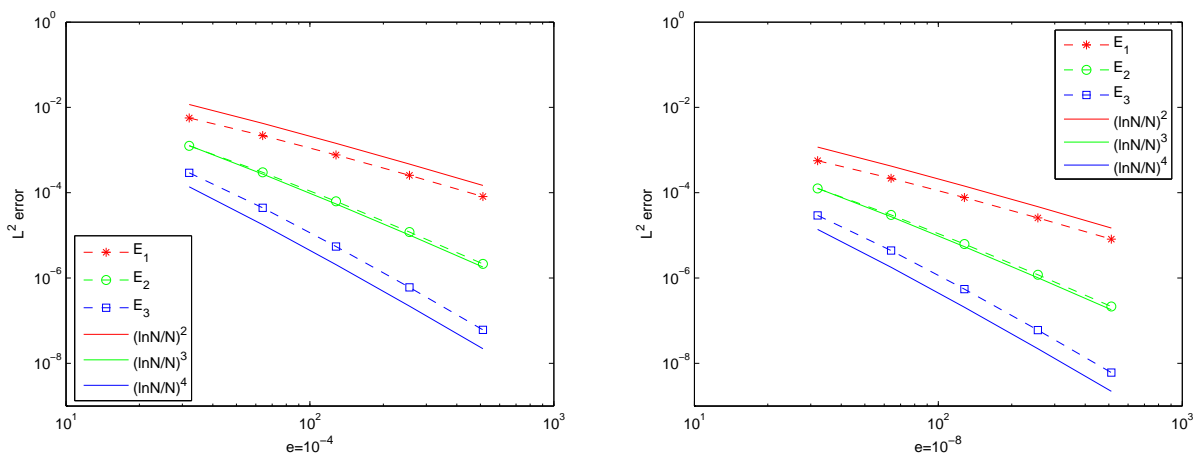

FIG. 5.3. The LDG method for Test Problem 3 with $k=1,2,3, \epsilon=10^{-4}, 10^{-8}$. 


\begin{tabular}{|c|cc|cc|cc|}
\hline & \multicolumn{7}{|c|}{$\epsilon=10^{-4}$} \\
\hline$N$ & $E_{1}$ & $r_{1}$ & $E_{2}$ & $r_{2}$ & $E_{3}$ & $r_{3}$ \\
\hline 32 & $5.64 \mathrm{e}-03$ & - & $1.26 \mathrm{e}-03$ & - & $2.93 \mathrm{e}-04$ & - \\
\hline 64 & $2.18 \mathrm{e}-03$ & 1.86 & $3.01 \mathrm{e}-04$ & 2.80 & $4.42 \mathrm{e}-05$ & 3.71 \\
\hline 128 & $7.70 \mathrm{e}-04$ & 1.93 & $6.28 \mathrm{e}-05$ & 2.91 & $5.48 \mathrm{e}-06$ & 3.87 \\
\hline 256 & $2.56 \mathrm{e}-04$ & 1.97 & $1.20 \mathrm{e}-05$ & 2.96 & $6.02 \mathrm{e}-07$ & 3.95 \\
\hline 512 & $8.15 \mathrm{e}-05$ & 1.99 & $2.15 \mathrm{e}-06$ & 2.99 & $6.09 \mathrm{e}-08$ & 3.98 \\
\hline \hline \multicolumn{7}{|c|}{$\epsilon=10^{-8}$} \\
\hline$N$ & $E_{1}$ & $r_{1}$ & $E_{2}$ & $r_{2}$ & $E_{3}$ & $r_{3}$ \\
\hline 32 & $5.65 \mathrm{e}-04$ & - & $1.26 \mathrm{e}-04$ & - & $2.93 \mathrm{e}-05$ & - \\
\hline 64 & $2.18 \mathrm{e}-04$ & 1.86 & $3.01 \mathrm{e}-05$ & 2.80 & $4.42 \mathrm{e}-06$ & 3.71 \\
\hline 128 & $7.70 \mathrm{e}-05$ & 1.93 & $6.28 \mathrm{e}-06$ & 2.91 & $5.48 \mathrm{e}-07$ & 3.87 \\
\hline 256 & $2.56 \mathrm{e}-05$ & 1.97 & $1.20 \mathrm{e}-06$ & 2.96 & $6.02 \mathrm{e}-08$ & 3.95 \\
\hline 512 & $8.15 \mathrm{e}-06$ & 1.99 & $2.15 \mathrm{e}-07$ & 2.99 & $6.09 \mathrm{e}-09$ & 3.98 \\
\hline
\end{tabular}

TABLE 5.3. History of convergence for $\left|\left(e_{q}, e_{u}\right)\right|_{\epsilon}$ for Test Problem 3. $\tau=(k+1) \sqrt{\epsilon} \ln N$.

\section{Concluding remarks}

In this paper, we present a priori error analysis of the LDG method on Shishkin meshes for one-dimensional singularly perturbed problems of convection-diffusion type and reaction-diffusion type. For both problems, we have proven that the orders of convergence of the LDG approximation are almost optimal and uniform regardless of the size of the perturbation parameter $\epsilon$. For the reaction-diffusion problem, the orders of convergence contain a positive power of parameter $\epsilon$, which has no negative impact on the convergence rates. Numerical experiments verify our theoretical finding. The analysis of the LDG method on other types of layer adapted meshes is currently underway. For two-dimensional problems, the superconvergence of the LDG method is discussed for the convection-diffusion problems with $\epsilon=1$ in [8]. We will consider the LDG approximation for two-dimensional singularly perturbed problems in a forthcoming paper.

Acknowledgments. The authors would like to thank the anonymous referees for their helpful remarks.

\section{REFERENCES}

[1] D.N. Arnold, F. Brezzi, B. Cockburn, and L.D. Marini, Unified analysis of discontinuous Galerkin methods for elliptic problems, SIAM J. Numer. Anal., 39, 1749-1779, 2002.

[2] F. Brezzi, B. Cockburn, L.D. Marini, and E. Süli, Stabilization mechanisms in discontinuous Galerkin finite element methods, Comp. Meth. Appl. Mech. Eng., 195, 3293-3310, 2006.

[3] F. Bassi and S. Rebay, A high-order accurate discontinuous finite element method for the numerical solution of the compressible Navier-Stokes equations, J. Comput. Phys., 131, 267-279, 1997.

[4] P. Castillo, B. Cockburn, D. Schötzau, and C. Schwab, Optimal a priori error estimates for the hp-version of the local discontinuous Galerkin method for convection-diffusion problems, Math. Comput., 71, 455-478, 2002.

[5] F. Celiker and B. Cockburn, Superconvergence of the numerical traces of discontinuous Galerkin and hybridized methods for convection-diffusion problems in one space dimension, Math. Comput., 76(257), 67-96, 2007.

[6] L. Chen and J. Xu, An optimal streamline diffusion finite element method for a singularly 
perturbed problem, Recent Advances in Adaptive Computation, Contemp. Math., 383, 91$201,2005$.

[7] Y. Cheng and C.W. Shu, Superconvergence of discontinuous Galerkin and local discontinuous Galerkin schemes for linear hyperbolic and convection-diffusion equations in one space dimension, SIAM J. Numer. Anal., 47, 4044-4072, 2010.

[8] B. Cockburn, G. Kanschat, I. Perugia, and D. Schötzau, Superconvergence of the local discontinuous Galerkin method for elliptic problems on Cartesian grids, SIAM J. Numer. Anal., 39(1), 264-285, 2001.

[9] B. Cockburn and C.W. Shu, The local discontinuous Galerkin finite element method for timedependent convection-diffusion systems, SIAM J. Numer. Anal., 35, 2440-2463, 1998.

[10] B. Cockburn and C.W. Shu, Runge-Kutta discontinuous Galerkin methods for convectiondominated problems, J. Sci. Comput., 16, 173-261, 2001.

[11] C. Johnson and J. Pitkäranta, An analysis of the discontinuous Galerkin method for a scalar hyperbolic equation, Math. Comput., 46, 1-26, 1986.

[12] P. Lesiant and P.A. Raviart, On a finite element method for solving the neutron transport equation, in Mathematical Aspects for Finite Elements in Partial Differential Equations (ed. C. de Boor), Academic Press, New York, 1974.

[13] R. Lin, Discontinuous discretization for least-squares formulation of singularly perturbed reaction-diffusion problems in one and two dimensions, SIAM J. Numer. Anal., 47(1), 89-108, 2008.

[14] R. Lin, A robust finite element method for singularly perturbed convection-diffusion problems, Disc. Cont. Dyn. Sys., 496-505, 2009.

[15] J.J. Miller, E. O'Riordan, and G.I. Shishkin, Fitted Numerical Methods for Singularly Perturbed Reaction-Diffusion Problems in Two and One Dimensions, World Scientific, Singapore, 1996.

[16] W.H. Reed and T.R. Hill, Trianglar mesh methods for the neutron transport equation, Technical Report LA-UR-73-479 Los Alamos Scientific Laboratory, Los Alamos, 1973.

[17] H.G. Roos, M. Stynes, and L. Tobiska, Robust Numerical Methods for Singularly Perturbed Differential Equations, Springer Series in Computational Mathematics, Springer-Verlag, 24, 2008.

[18] H.G. Roos and H. Zarin, A supercloseness result for the discontinuous Galerkin stabilization of convectiondiffusion problems on Shishkin meshes, Numer. Meth. Par. Diff. Eq., 23(6), 1560-1576, 2007.

[19] D. Schötzau and C. Schwab, Time discretization of parabolic problems by the hp-version of the discontinuous Galerkin finite element method, SIAM J. Numer. Anal., 38, 837-875, 2000.

[20] Z. Xie and Z. Zhang, Superconvergence of DG method for one-dimensional singularly perturbed problems, J. Comput. Math., 25(2), 185-200, 2007.

[21] Z. Xie and Z. Zhang, Uniform superconvergence analysis of the discontinuous Galerkin method for a singularly perturbed problem in 1-D, Math. Comput., 79(269), 35-45, 2010.

[22] Z. Xie, Z. Zhang, and Z. Zhang, A numerical study of uniform superconvergence of LDG method for solving singularly perturbed problems, J. Comput. Math., 27(2), 280-298, 2009.

[23] Z. Zhang, Finite element superconvergence approximation for one-dimensional singularly perturbed problems, Numer. Meth. Part. Diff. Equs., 18(3), 374-395, 2002.

[24] H. Zhu, Discontiunous Galerkin Methods for Singularly Perturbed Problems, Ph.D. thesis, Wayne State University, Detroit, 2009. 